\title{
On the Performance Analysis of Wireless Receiver in Cascaded Fading Channel
}

\author{
Jyoteesh Malhotra \\ Department of Electronics and Communication Engineering \\ G.N.D.U. Regional Campus \\ Jalandhar, India \\ jyoteesh@rediffmail.com
}

\author{
Ajay K. Sharma \\ Department of Computer Science and Engineering \\ National Institute of Technology \\ Jalandhar, India \\ sharmaajayk@rediffmail.com
}

\author{
R.S.Kaler \\ Department of Electronics and Communication Engineering \\ Thapar University \\ Patiala, India \\ rskaler@yahoo.com
}

\begin{abstract}
In this paper, we provide a unified analysis for wireless system over cascaded fading channels modeled either by cascaded Nakagami-m or Weibull fading models. These cascade fading models are developed by the product of independent Nakagami-m or Weibull random variables, which are not necessary identically distributed. The performance measures such as amount of fading, average bit error rate, and signal outage are computed in this analysis. We first use the Padé approximation (PA) technique to find simple to evaluate rational expressions for the moment generating function (MGF) of output signal-to-noise ratio (SNR), unlike previously derived intricate expressions in terms of MeijerG function for cascaded Nakagami-m fading channel. Rational expressions for the MGF of the cascaded Weibull random variable are also computed to provide new set of performance results. Using these rational expressions, we analyze the performance of wireless receivers under a range of representative channel fading conditions using both cascaded fading models. To verify the correctness of the proposed rational expression formulation numerical and computer simulations has been done, which shows perfect match.
\end{abstract}

Keywords-Keyhole Channels, Multiple-Input Multiple-output (MIMO), Nakagami-m distribution, Weibull Distribution, Outage probability, Bit error probability.

\section{INTRODUCTION}

Wireless systems suffer from problems introduced by the radio propagation which is characterized by three nearly independent phenomena: path loss variance with distance, shadowing (or long-term fading), and multipath (or short-term) fading. Except path loss, which is only distance dependent, the other two phenomena can be statistically described by fading models with parameters determined using experimental measurements. Depending on the radio propagation environment and underlying communication scenario, there is range of statistical multipath fading models available in the literature [1]. For long-term fading conditions, it is widely accepted that the probability density function (PDF) of the fading envelopes can be modeled by the lognormal distribution [1]. Due to ever-increasing demand and ubiquitous access of personal communication services, wireless systems are required to operate in increasingly hostile environments. As expectations for the performance and reliability of wireless systems become more demanding, the significance of accurate channel modeling in system design, evaluation, and deployment will continue [2]. Short-term fading models include the well-known Rayleigh, Rice [3], Hoyt [4], and Nakagami-m [5] distributions. Lately, Weibull statistical distribution [6], [7] has been found to fit well with experimental short-term fading for mobile communications. Recently, cascaded fading model has been used [8], which do not separate the fading in several parts but rather study the phenomenon as a whole. The so-called cascaded Rayleigh (i.e. Rayleigh x Rayleigh) fading stochastic model has been found to be suitable when large number of rays are reflected by two independent scatters and both transmitter \& receiver are moving [9]. The double cascade Rayleigh model has also been recently used for keyhole channel modeling of MIMO systems [10]. Extending this model by characterizing the fading between each pair of transmit and receive antennas in the presence of the keyhole as Nakagami-m, the double cascaded Nakagami-m (Nakagami-m x Nakagami-m) fading model has also been considered in [11]. Such products of random variables have also been found to be useful for analyzing the performance of multihop systems [12]. In an effort to generalize the research work relate to cascaded fading mentioned earlier, the $\mathrm{N}$ x Nakagami-m distribution developed as the product of $\mathrm{N}$ statistically independent, but not necessarily identically distributed, Nakagami-m RVs in [13]. In a similar generalization cascaded Weibull fading channel model has been developed in [14]. The closed form expressions of cumulative distribution function (CDF) and MGF in terms of MeijerG function were presented in [13] to compute outage probability and average bit error probability (ABEP), respectively. The closed form expression for PDF of cascaded Weibull fading channel model and channel capacity have also been derived in terms of MeijerG function in [14]. These closed form expressions despite having an elegant form, suffer from a major drawback. Although, MeijerG special function used can be evaluated in itself using symbolic mathematical packages such as Mathematica \& Maple, but integrals using 
this special function lead to numerical instabilities and erroneous results at higher shaping parameter values. This renders the expressions impractical from the ease of computation point of view. Thus, a unified and easy to compute performance analysis for the SNR statistics of a receiver operating over Cascaded fading channel is required. Moreover, the signal outage and ABEP analysis of cascaded Weibull fading channel is not available in open literature, because it is still not possible to derive the closed form expression of its MGF. In this paper, moments-based PA technique has been used to obtain simple to evaluate rational expressions for the MGF of Cascaded Nakagami-m and Weibull RVs. Using these novel expressions, we study the signal outage and ABEP of important digital modulation schemes for receiver operating on both cascaded Weibull and Nakagami-m fading channels. Earlier, the PA technique was used for performance analysis of diversity systems in Nakagami-m fading [15] and more recently in Weibull fading channels [16]. In this analysis, the effect of fading severity on the performance is investigated in both cascaded Nakagami-m \& Weibull fading channels. Computer simulations are also generated for the result verifications.

The remaining of the paper is organized as follows. In the following section, we present our system \& channel model. Section III details the performance analysis of the system in terms of amount of fading, ABEP and outage probability using both the models. Numerical and simulation results are presented in Section IV before the paper is finally concluded in Section V.

\section{SyStem AND CHANNEL MODEL}

We consider signal transmission over cascaded fading channels. The baseband representation of the received signal is given by $z=s Y+n$ where $s$ is the transmitted baseband symbol which can take different values from modulation alphabets such as M-quadrature amplitude modulation (MQAM) and M-phase shift keying (MPSK), $Y$ represents slow, frequency-flat channel's cascade-faded envelope which is either $N$ x Nakagami or $N$ x Weibull distributed, and $n$ is the additive white Gaussian noise (AWGN). We define the distribution of $Y$ as the product of $N$ independent, but not necessarily identically distributed (i.n.d.) RVs $R_{l}$.

$Y=\prod_{l=1}^{N} R_{l}$

Using (1), the nth order moment of ' $Y$ ' can be expressed

$E\left[Y^{n}\right]=E\left[\prod_{l=1}^{N} R_{l}^{n}\right]$

Since the RVs $R_{l}$ are mutually independent, the above equation can be expressed as the product of the nth order moments of each RV.

$E\left[Y^{n}\right]=\prod_{l=1}^{N} E\left[R_{l}^{n}\right]$
Let us consider a transmission over the previously described cascade-faded channel, and in the presence of AWGN. The instantaneous SNR per symbol at the input of its receiver is given by [1]

$\gamma=\frac{E_{s}}{N_{o}} Y^{2}$

where $E_{s}$ is the transmitted symbol's average energy and $N_{0}$ is the single-sided AWGN power spectral density. The corresponding average SNR is

$\bar{\gamma}=\frac{E_{s}}{N_{0}} E\left[Y^{2}\right]=\frac{E_{s}}{N_{0}} \prod_{l=1}^{N} E\left[R_{l}^{2}\right]$

Now, the nth moment of $\gamma$ can be easily derived as

$E\left[\gamma^{n}\right]=\frac{E_{s}}{N_{0}} \prod_{l=1}^{N} E\left[\left(R_{l}^{2}\right)^{n}\right]$

\section{A. Cascaded Weibull Fading Model}

Let us consider $N \geq 1$ Weibull distributed (i.n.d.) RVs each with PDF given as

$$
f_{R_{l}}(r)=\frac{c_{l}}{\Omega_{l}} r^{c_{l}-1} \exp \left\{-\frac{r^{c_{l}}}{\Omega_{l}}\right\} \quad r \geq 0
$$

where $c_{l}$ is the shape parameter and $\Omega_{l}$ is related to the mean square value of $\mathrm{RV}$ as

$$
E\left[R_{l}^{2}\right]=\Omega_{l}^{\frac{2}{c_{l}}} \Gamma\left(1+\frac{2}{c_{l}}\right)
$$

where $\Gamma($.$) is the gamma function. The nth order moment$ of squared $\mathrm{RV}$ is given as [1]

$$
E\left[\left(R_{l}^{2}\right)^{n}\right]=\Omega_{l}^{\frac{2 n}{c_{l}}} \Gamma\left(1+\frac{2 n}{c_{l}}\right)
$$

With the support of (5) and (6), the nth moment of SNR in cascaded Weibull fading channel is given as

$$
E\left[\gamma^{n}\right]=\bar{\gamma}^{n} \prod_{l=1}^{N} \frac{\Gamma\left(1+\frac{2 n}{c_{l}}\right)}{\Gamma^{n}\left(1+\frac{2}{c_{l}}\right)}
$$




\section{B. Cascaded Nakagami-m Fading Model}

Let us consider $N \geq 1$ Nakagami-m distributed (i.n.d.) RVs each with PDF given as

$$
f_{R_{l}}(r)=\frac{2 m_{l}^{m_{l}}}{\Omega_{l}^{m_{l}} \Gamma\left(m_{l}\right)} r^{2 m_{l}-1} \exp \left\{-\frac{m_{l} r^{2}}{\Omega_{l}}\right\} \quad r \geq 0
$$

where $m_{l}$ is the shape parameter and $\Omega_{l}$ is the mean square value of RV.

The nth order moment of squared $\mathrm{RV}$ is given as

$$
E\left[\left(R_{l}^{2}\right)^{n}\right]=\frac{\Gamma\left(m_{l}+n\right)}{\Gamma\left(m_{l}\right) m_{l}{ }^{n}}\left(E\left[R_{l}^{2}\right]\right)^{n}
$$

Using (5) and (6), the nth moment of output SNR in cascaded Nakagami-m fading channel is given as

$$
E\left[\gamma^{n}\right]=\bar{\gamma}^{n} \prod_{l=1}^{N} \frac{\Gamma\left(m_{l}+n\right)}{\Gamma\left(m_{l}\right) m_{l}^{n}}
$$

\section{Performance ANALysis}

In order to quantify the performance in terms of ABEP and signal outage, well known MGF based unified approach [1] will be used. We will use moments-based PA technique to find simple to evaluate rational expressions for the MGF as follows.

The MGF of an RV $\gamma>0$ is

$M_{\gamma}(s)=E\left[e^{-s x}\right]=\int_{0}^{\infty} e^{-s \gamma} f_{\gamma}(\gamma) d \gamma$

It is interesting to note that the nth moments of the instantaneous SNR statistics available in closed-form for both cascaded Weibull and Nakagami-m fading channels. Using the Taylor series expansion of $e^{-s \gamma}$, the MGF given by (14) can be expressed in terms of a power series as

$$
\begin{aligned}
M_{\gamma}(s) & =\sum_{n=0}^{\infty} \frac{(-1)^{n}}{n !} E\left[\gamma^{n}\right] s^{n} \\
& =\sum_{n=0}^{\infty} b_{n} s^{n}
\end{aligned}
$$

where $b_{n}=\frac{(-1)^{n}}{n !} \bar{\gamma}^{n} \prod_{l=1}^{N} \frac{\Gamma\left(1+2 n / c_{l}\right)}{\Gamma^{n}\left(1+2 / c_{l}\right)}$ for cascaded Weibull fading channel and $b_{n}=\frac{(-1)^{n}}{n !} \bar{\gamma}^{n} \prod_{l=1}^{N} \frac{\Gamma\left(m_{l}+n\right)}{\Gamma\left(m_{l}\right) m_{l}{ }^{n}}$ for cascaded Nakagami-m fading channel.

The infinite series in (15) is not guaranteed to converge for all values of $s$. But it is possible, using PA technique to obtain efficiently the limiting behavior of a power series in compact rational function form $[17,18]$. In particular, the one-point PA of order $(D-1 / D)$ is defined from the series $(15)$ in a rational function form by

$M_{\gamma}(s) \square \frac{\sum_{i=0}^{D-1} a_{i} s^{i}}{\sum_{j=0}^{D} b_{j} s^{j}}$

where $a_{i}$ and $b_{j}$ are the coefficients such that

$\frac{\sum_{i=0}^{D-1} a_{i} s^{i}}{\sum_{j=0}^{D} b_{j} s^{j}}=\sum_{n=0}^{2 D-1} c_{n} s^{n}+O\left(s^{2 D}\right)$

where $O\left(s^{2 D}\right)$ representing the terms of order higher than $2 D-1$. The coefficients $b_{j}$ can be found using (assuming $b_{0}=1$ ) following equations

$\sum_{j=0}^{D} b_{j} c_{D-1-j+d}=0 \quad 0 \leq d \leq D$

The above equations form a system of $D$ linear equations for the unknown denominator coefficients in (16). This system of equations can be uniquely solved, as long as the determinant of its Hankel matrix is nonzero [17]. The choice of the value of $D$ is indeed a critical issue, as it represents a tradeoff between the accuracy of the PA technique and the complexity of the system of equations to be solved. It is described in [17] that there exist a value of $D$ above which Hankel matrix become rank deficient. After solving for the values of $b_{j}$, the numerator coefficients $a_{i}$ can now be obtained using

$a_{i}=c_{i}+\sum_{p=1}^{\min (D, i)} b_{i} c_{i-p}=0 \quad 0 \leq i \leq D-1$

Having obtained the coefficients of denominator and numerator polynomials, an appropriate expression for the MGF of the output SNR is now available in rational function form. We are now ready to present three important performance measures namely, the Amount of Fading, the ABEP for different modulation schemes and outage probability in the Cascaded fading channels.

\section{A. Amount of Fading}

The Amount of Fading $(\mathrm{AoF})$ is an important performance measure of the fading channel. This moments-based, dimensionless parameter characterizes the severity of the fading channel and can be easily computed from first and second moments as [1]

$$
A o F=\frac{E\left[\gamma^{2}\right]}{\bar{\gamma}^{2}}-1
$$


Using (10), AoF for cascaded Weibull fading channel is given as

$$
A o F=\prod_{l=1}^{N}\left(\frac{\Gamma\left(1+4 / c_{l}\right)}{\Gamma^{2}\left(1+2 / c_{l}\right)}\right)-1
$$

Using (13), AoF for cascaded Nakagami-m fading channel is given as

$$
A o F=\prod_{l=1}^{N}\left(1+\frac{1}{m_{l}}\right)-1
$$

Figure $1 \& 2$ depicts the AoF plotted as a function of fading parameters in cascaded Weibull and Nakagami-m fading channels, respectively for different values of $N$. It can be observed that at a fixed value of fading parameter the severity of fading increases for higher cascading number $N$. As seen in figures, the $A o F$ in cascaded Weibull fading channel increases abruptly than cascaded Nakagami-m fading channels with increase in $N$.

\section{B. Average Bit Error Probability}

\section{1) M-Quadrature Amplitude Modulation(MQAM)}

In the single channel receiver, the conditional BEP of Gray encoded MQAM using alternative Gaussian- $Q$ function form in [1], is given as

$$
\begin{aligned}
P_{e}(\gamma) & =\frac{4}{\pi \log _{2}(M)}\left(1-\frac{1}{\sqrt{M}}\right) \times \\
& \left\{\int_{0}^{\pi / 2} \exp \left(-\frac{g_{q a m}}{\sin ^{2}(\phi)} \gamma\right) d \phi\right. \\
& \left.-\int_{0}^{\pi / 4} \exp \left(-\frac{g_{q a m}}{\sin ^{2}(\phi)} \gamma\right) d \phi\right\}
\end{aligned}
$$

where $g_{\text {qam }}=3 / 2(M-1), M$ represents the number of symbols and $\gamma$ is the instantaneous SNR per symbol.

Averaging over the PDF of the received SNR the ABEP becomes

$$
\begin{aligned}
\bar{P}_{e}= & \frac{4}{\pi \log _{2}(M)}\left(1-\frac{1}{\sqrt{M}}\right) \times \\
& \left\{\int_{0}^{\pi / 2} M_{\gamma}\left(-\frac{g_{\text {qam }}}{\sin ^{2}(\phi)}\right) d \phi\right. \\
& \left.-\int_{0}^{\pi / 4} M_{\gamma}\left(-\frac{g_{\text {qam }}}{\sin ^{2}(\phi)}\right) d \phi\right\}
\end{aligned}
$$

where $M_{\gamma}($.$) represents the MGF of Cascaded Weibull or$ Nakagami-m RV.

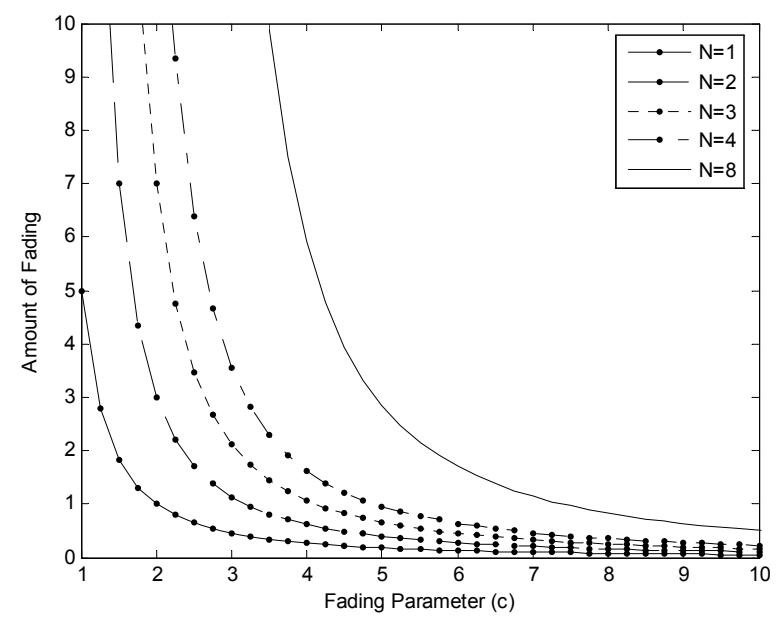

Figure 1. Amount of Fading as a function of $c$ for several values of $\mathrm{N}$.

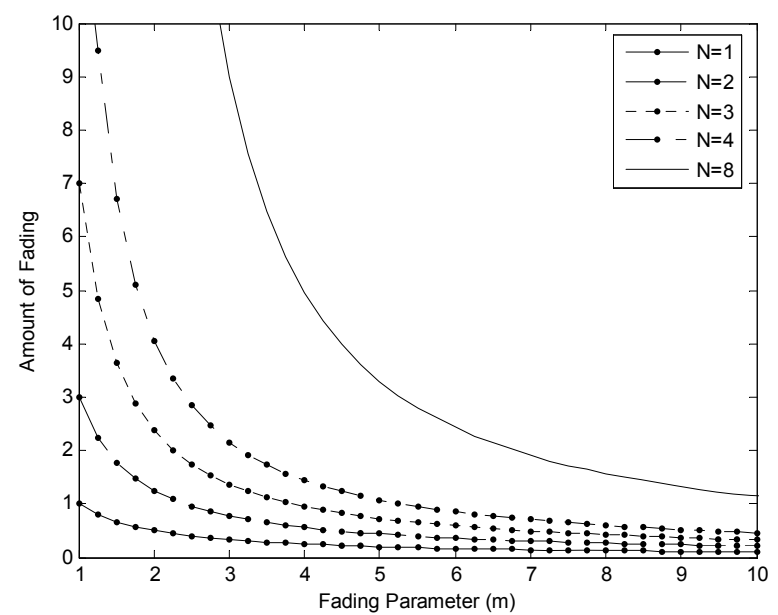

Figure 2. Amount of Fading as a function of $m$ for several values of $\mathrm{N}$.

\section{2) M-Phase Shift Keying(MPSK)}

In the single channel receiver, the conditional bit error probability of Gray encoded MPSK using alternative Gaussian$\mathrm{Q}$ function form in [1], is given as

$P_{e}(\gamma) \cong \frac{1}{\pi \log _{2}(M)} \int_{0}^{(M-1) \pi / M} \exp \left(\frac{g_{p s k}}{\sin ^{2}(\phi)} \gamma\right) d \phi$

where $g_{p s k}=\sin ^{2}\left(\frac{\pi}{M}\right)$. Averaging over the PDF of the received SNR the ABEP becomes

$$
\bar{P}_{e} \cong \frac{1}{\pi \log _{2}(M)} \int_{0}^{(M-1) \pi / M} M_{\gamma}\left(\frac{g_{p s k}}{\sin ^{2}(\phi)}\right) d \phi
$$




\section{Outage Probability}

The signal outage probability is defined as the probability that the instantaneous SNR falls below a certain threshold, $\gamma_{t h}$ i.e.

$$
P_{\text {out }}\left(\gamma_{\text {th }}\right) \square P\left(S N R<\gamma_{\text {th }}\right)
$$

For single channel receiver, using MGF approach [1, Chap. 1] the outage probability can be computed as

$$
P_{\text {out }}\left(\gamma_{\text {th }}\right)=\frac{1}{2 \pi j} \int_{\varepsilon-j \infty}^{\varepsilon+j \infty} \frac{M_{\gamma}(s)}{s} e^{s \gamma_{\text {th }}} d s
$$

where $\varepsilon$ is a properly chosen constant in the region of convergence of complex s-plane. Interestingly, since $M_{\gamma}(s)$ is given in terms of a rational function form, one can use the partial fraction expansion of $\left[M_{\gamma}(s)\right] / s$ in (28) to evaluate outage probability, i.e.

$$
\begin{aligned}
P_{\text {out }}\left(\gamma_{\text {th }}\right)=\frac{1}{2 \pi j} \int_{\varepsilon-j \infty}^{\varepsilon+j \infty} \sum_{i=1}^{N_{p}} \frac{\lambda_{i}}{s+p_{i}} e^{s \gamma_{\text {th }}} d s \\
=\frac{1}{2 \pi j} \sum_{i=1}^{N_{p}} \int_{\varepsilon-j \infty}^{\varepsilon+j \infty} \frac{\lambda_{i}}{s+p_{i}} e^{s \gamma_{\text {th }}} d s \\
=\sum_{i=1}^{N_{p}} \lambda_{i} e^{-p_{i} \gamma_{\text {th }}}
\end{aligned}
$$

where $p_{i}$ are the $N_{p}$ poles of rational function in $s$ with $\lambda_{i}$ its residues. Each term inside the summation in (29) represents a simple exponential closed form function and can be easily evaluated.

Clearly, using the rational function for the MGF provided by the PA technique, the integrals in (24) and (26) can be easily evaluated numerically and the results are found to be very stable. In fact some of the integrals, like the one in (28), closed form can be found as it is equivalent to the problem of finding the inverse Laplace transform of a rational function, which can be easily solved using the partial fractions expansion as in (29).

\section{Numerical AND SimUlation Results}

We compute the rational expressions using PA technique of order $<9 / 10>\&<15 / 16>$ for Cascaded Nakagami-m fading and Weibull fading model, respectively. Table I lists the $\left\{a_{i}\right\}$ and $\left\{b_{j}\right\}$ coefficient sets for the rational function form of MGF for different values of $N$ and $m$, representing various cascaded Nakagami-m fading channel conditions. The numerator and denominator coefficients of rational expressions (MGF) of cascaded Weibull fading are not tabulated for brevity. Hence, PA technique leads to rational expressions which are computationally simple for analysis. ABEP of digital modulations and Outage Probability through cascaded fading channel have been numerically evaluated using these rational functions and compared for accuracy with simulation results.

\section{A. Average BEP of Digital Modulations}

We have chosen four illustrative examples for performance evaluation of the wireless receiver in terms of ABEP. The first is depicted in figure 3 for the case of 16-PSK in cascaded Nakagami-m fading channel, and second in figure 4 for the case of 16-PSK in cascaded Weibull fading channel, both the two cases versus the average SNR. Computer simulation of ABEP for the eight representative channel fading conditions in each of $(N=1, m=4.5 ; N=1, m=9.5 ; N=2, m=4.5 ; N=2$, $m=9.5 ; N=3, m=4.5 ; N=3, m=9.5 ; N=4, m=4.5 ; N=4$, $m=9.5)$ cascaded Nakagami-m, and $(N=1, c=4.5 ; N=1, c=$ 9.5; $N=2, c=4.5 ; N=2, c=9.5 ; N=4, c=4.5 ; N=4, c=$ 9.5; $N=8, c=4.5 ; N=8, c=9.5$ ) cascaded Weibull model is obtained and compared with results evaluated using rational expressions for similar channel conditions. In figure $5 \& 6$ ABEP performance of 16-QAM is evaluated in various cascaded Nakagami-m and Weibull channel fading condition as mentioned earlier. It is evident from figures that the ABEP improves as average SNR $(\bar{\gamma})$ increases and for a fixed value of $\bar{\gamma}$ also, ABER improves with an increase of $m$ or $c$. As depicted the results obtained using rational expressions and simulations shows perfect agreement. The analysis based on MeijerG function was done in [14] but ABEP and outage probability results are not available for cascaded Weibull fading channel. Also, the modern software packages such as Mathematica and Maple fail to handle the integrals involving MeijerG function, especially the higher values of fading parameters $m$ or $c$ leads to numerical instabilities and erroneous results.

TABLE I. NUMERATOR AND DENOMINATOR COEFFICIENTS OF RATIONAL EXPRESSIONS OF MGF (CASCADED NAKAGAMI FADING).

\begin{tabular}{|c|c|c|l|l|}
\hline $\boldsymbol{N}$ & $\boldsymbol{m}$ & Order & \multicolumn{1}{|c|}{$\begin{array}{c}\text { Numerator Coefficients }\{\mathbf{a}\} \\
\left(\boldsymbol{a}_{\boldsymbol{o}}=\mathbf{1}\right)\end{array}$} & \multicolumn{1}{c|}{$\begin{array}{c}\text { Denominator Coefficients }\{\mathbf{b}\} \\
\left(\boldsymbol{b}_{\boldsymbol{o}}=\mathbf{1}\right)\end{array}$} \\
\hline 2 & 4.5 & $<\mathbf{6 / 7}>$ & $\{4.65,7.82,5.52,1.37,-0.023,0.35 \mathrm{e}-3\}$ & $\{5.65,12.7,14.5,8.97,2.92,0.44,0.23 \mathrm{e}-1\}$, \\
\hline 2 & 6.3 & $<\mathbf{6 / 7}>$ & $\{2.2,1.54,0.33,-0.11 \mathrm{e}-1,0.31 \mathrm{e}-3,-0.68 \mathrm{e}-5\}$ & $\{3.2,4.07,2.64,0.93,0.17,0.15 \mathrm{e}-1,0.49 \mathrm{e}-3\}$ \\
\hline 2 & 7.5 & $<\mathbf{6 / 7}>$ & $\{1.64,0.84,0.12,-0.77,0.3 \mathrm{e}-3,-0.63 \mathrm{e}-5\}$ & $\{2.64,2.84,1.6,0.51,0.91 \mathrm{e}-1,0.84 \mathrm{e}-2,0.32 \mathrm{e}-3\}$ \\
\hline 4 & 4.5 & $<\mathbf{9 / 1 0}>$ & $\{151,8.5 \mathrm{e} 3,2.3 \mathrm{e} 5,3.2 \mathrm{e} 6,2.4 \mathrm{e} 7,9.1 \mathrm{e} 7,1.6 \mathrm{e} 8,9 \mathrm{e} 7,-3.4 \mathrm{e} 6\}$ & $\{152,8.6 \mathrm{e} 3,2.4 \mathrm{e} 5,3.4 \mathrm{e} 6,2.7 \mathrm{e} 7,1.1 \mathrm{e} 8,2.5 \mathrm{e} 8,2.4 \mathrm{e} 8,8.8 \mathrm{e} 7,4.3 \mathrm{e} 6\}$ \\
\hline 4 & 6.3 & $<\mathbf{9 / 1 0}>$ & $\{58,1.3 \mathrm{e} 3,1.4 \mathrm{e} 4,8.2 \mathrm{e} 4,2.6 \mathrm{e} 5,4.4 \mathrm{e} 5,3.4 \mathrm{e} 5,8.8 \mathrm{e} 4,-3.4 \mathrm{e} 3\}$ & $\{59,1.3 \mathrm{e} 3,1.5 \mathrm{e} 4,9.7 \mathrm{e} 4,3.5 \mathrm{e} 5,7.1 \mathrm{e} 5,8.1 \mathrm{e} 5,4.9 \mathrm{e} 5,1.4 \mathrm{e} 5,1.4 \mathrm{e} 4\}$ \\
\hline 4 & 7.5 & $<\mathbf{9 / 1 0}>$ & $\{36,5 \mathrm{e} 2,3.4 \mathrm{e} 3,1.2 \mathrm{e} 4,2.4 \mathrm{e} 4,2.3 \mathrm{e} 4,8.8 \mathrm{e} 3,2.4 \mathrm{e} 2,21.8\}$ & $\{37,5.3 \mathrm{e} 2,3.9 \mathrm{e} 3,1.6 \mathrm{e} 4,3.7 \mathrm{e} 4,5 \mathrm{e} 4,3.7 \mathrm{e} 4,1.4 \mathrm{e} 4,2.4 \mathrm{e} 3,1.6 \mathrm{e} 2\}$ \\
\hline 8 & 4.5 & $<\mathbf{9 / 1 0}>$ & $\{5.9 \mathrm{e} 4,1 \mathrm{e} 9,5.9 \mathrm{e} 12,1.3 \mathrm{e} 16,1 \mathrm{e} 19,2.8 \mathrm{e} 21,2.3 \mathrm{e} 23,4.6 \mathrm{e} 24,1.6 \mathrm{e} 25\}$ & $\{5.9 \mathrm{e} 4,9.9 \mathrm{e} 8,5.9 \mathrm{e} 12,1.3 \mathrm{e} 16,1 \mathrm{e} 19,2.8 \mathrm{e} 21,2.3 \mathrm{e} 23,4.9 \mathrm{e} 24,2 \mathrm{e} 25,1.1 \mathrm{e} 25\}$ \\
\hline 8 & 6.3 & $<\mathbf{9 / 1 0}>$ & $\{8.1 \mathrm{e} 3,2 \mathrm{e} 7,1.8 \mathrm{e} 10,6.7 \mathrm{e} 12,1 \mathrm{e} 15,6 \mathrm{e} 16,1.3 \mathrm{e} 18,8.8 \mathrm{e} 18,1.4 \mathrm{e} 19\}$ & $\{8.1 \mathrm{e} 3,2 \mathrm{e} 7,1.8 \mathrm{e} 10,6.7 \mathrm{e} 12,1 \mathrm{e} 15,6.1 \mathrm{e} 16,1.3 \mathrm{e} 18,1 \mathrm{e} 19,2.3 \mathrm{e} 19,1.1 \mathrm{e} 19\}$ \\
\hline 8 & 7.5 & $<\mathbf{9 / 1 0}>$ & $\{2.96 \mathrm{e} 3,2.6 \mathrm{e} 6,8.9 \mathrm{e} 8,1.2 \mathrm{e} 11,5.7 \mathrm{e} 12,5.7 \mathrm{e} 13,-1.1 \mathrm{e} 15,-1.5 \mathrm{e} 16,-3.2 \mathrm{e} 16\}$ & $\{3 \mathrm{e} 3,2.6 \mathrm{e} 6,9 \mathrm{e} 8,1.2 \mathrm{e} 11,5.8 \mathrm{e} 12,6.3 \mathrm{e} 13,-1.1 \mathrm{e} 15,1.6 \mathrm{e} 16,-4.7 \mathrm{e} 16 .-2.8 \mathrm{e} 16\}$ \\
\hline
\end{tabular}




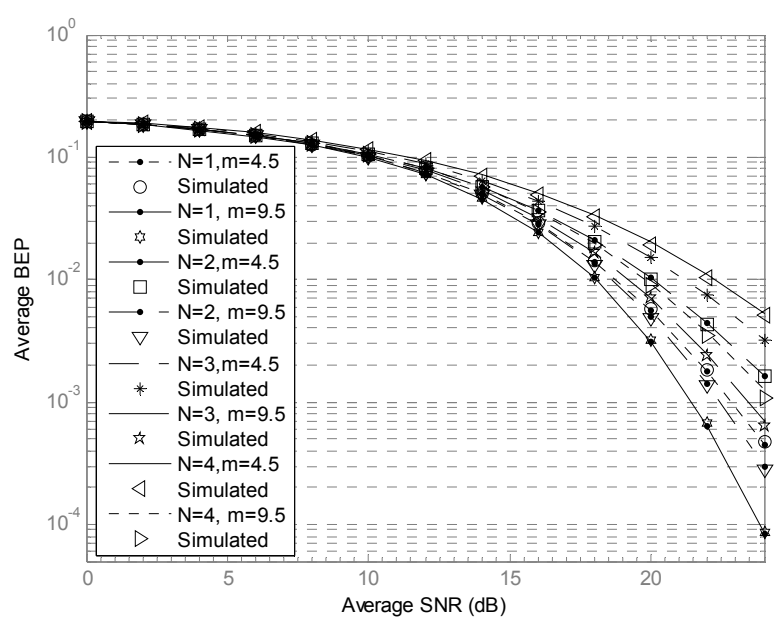

Figure 3. Average BER of 16-PSK versus average SNR in Cascaded Nakagami-m fading channel

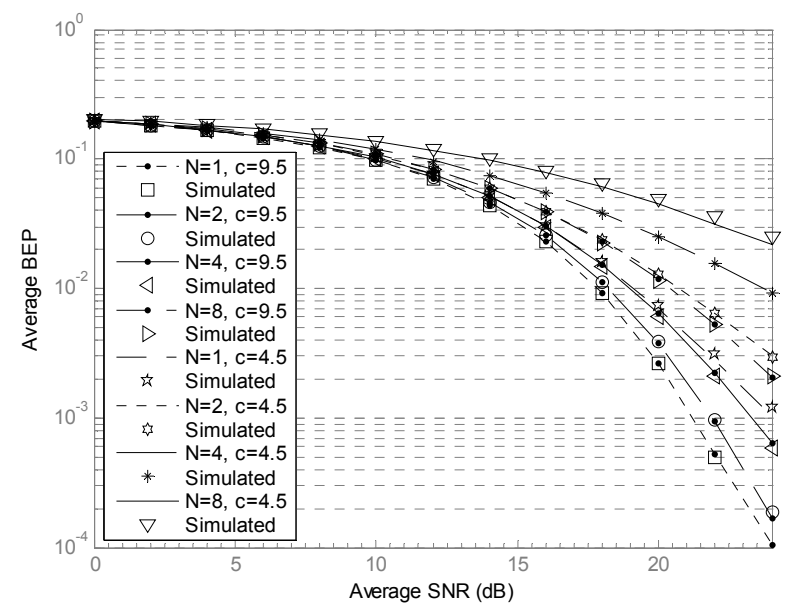

Figure 4. Average BER of 16-PSK versus average SNR in Cascaded Weibull fading channel.

Thus, moments-based approach provides an alternative simple to evaluate rational expressions of MGF which resulted in unified digital modulation analysis over cascaded fading channels.

\section{B. Outage Probability}

Figures $7 \& 8$ depicts the outage probability versus the threshold $\gamma_{\text {th }}$ normalized by scaling parameter $\bar{\gamma}$ in cascaded Nakagami-m and Weibull fading channels, respectively. The outage probability results are numerically evaluated using (29) and corroborated with Monte-Carlo simulations for both cascade fading models. The results shows that for fixed cascading level the outage probability decreases with increasing fading parameter values because fading severity of channel decreases. On the contrary, outage probability increases as the cascading number $N$ increases for the fixed fading parameters value $m$ or $c$. This is due to more deep fades that are generated by the product of $N$ random variables.

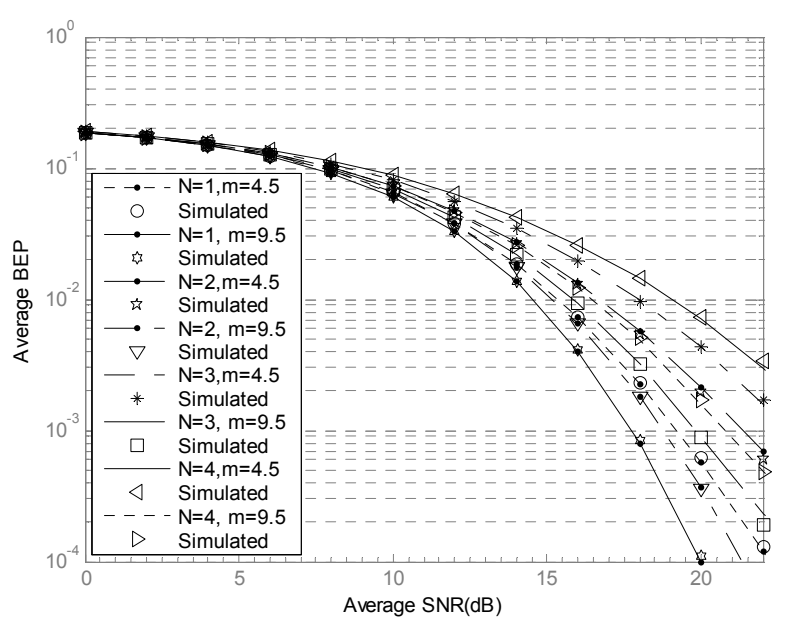

Figure 5. Average BER of 16-QAM versus average SNR in Cascaded Nakagami-m fading channel .

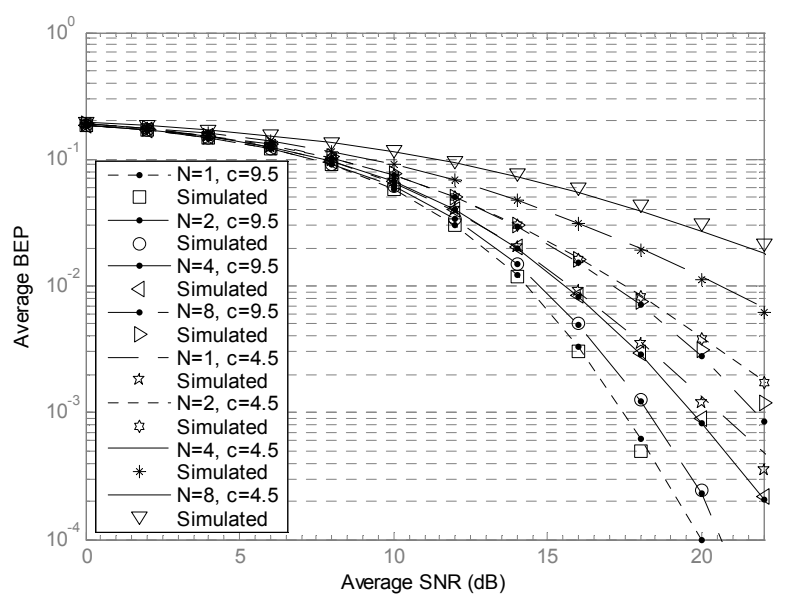

Figure 6. Average BER of 16-QAM versus average SNR in Cascaded Weibull fading channel.

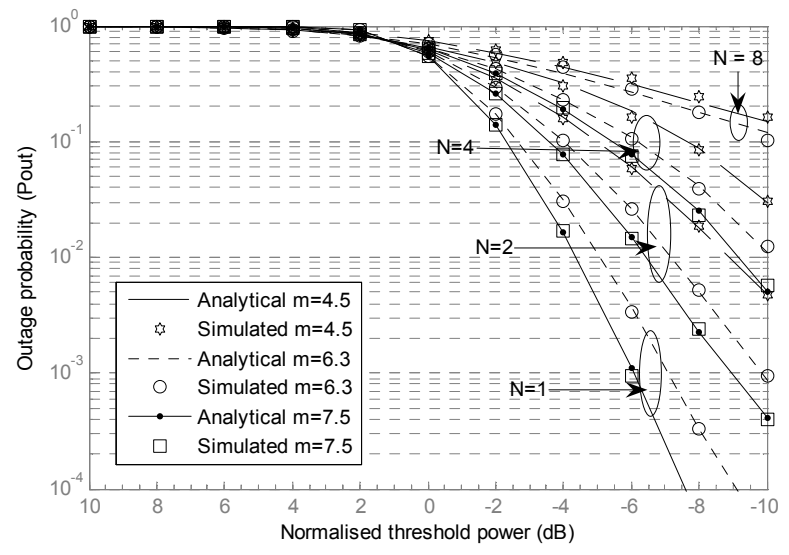

Figure 7. Outage probability vs. Normalized threshold in Cascaded Nakagami-m Fading Channel. 


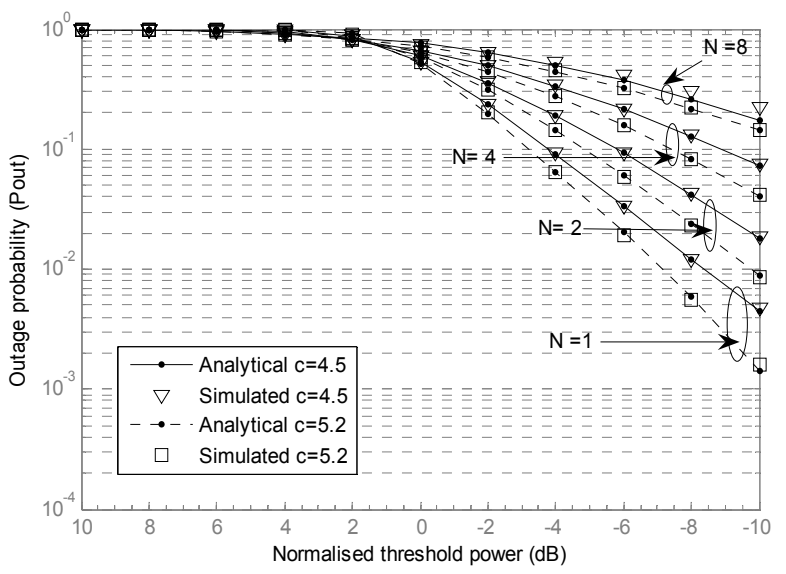

Figure 8. Outage probability vs. Normalized threshold in Cascaded Weibull Fading Channel .

From these plots, it is evident that rational expressions can be used to give very accurate estimate of the MGF for arbitrary values of $N, C$ and $m$ singly or collectively. Note that if the accuracy is not satisfactory for some cases, it is always possible to choose a higher value of $D$ to enhance accuracy as long as the Hankel matrix is not rank deficient.

\section{CONCLUSIONS}

The Cascaded fading channel can be modeled by multiplying independent Nakagami-m or Weibull RVs, which may not be identically distributed. These two fading models have been used to analyze the wireless system performance in terms of amount of fading, outage probability and average bit error probability through cascaded fading channels. In doing so, moments-based simple to evaluate rational expressions for the MGF of output SNR are obtained for both the models. The unified approach presented here not only simplified the performance analysis in cascaded Nakagami-m fading channels but also provided new performance results in cascaded Weibull fading channels. To compliment the theoretical content of the paper numerical and simulation results are also presented for different channel fading conditions. The results obtained from rational expressions and computer simulation shows perfect match.

\section{REFERENCES}

[1] M. K. Simon and M.-S. Alouini, Digital Communication Over Fading Channels, $2^{\text {nd }}$ ed. New York: Wiley, 2005.

[2] L. J. Greenstein, J. B. Andersen, H. L. Bertoni, S. Kozono, D. G. Michelson, and W. H. Tranter, "Channel and propagation models for wireless systems design," IEEE J. Sel. Areas Commun., vol. 20, no. 3, pp. 493- 495, Apr. 2002.

[3] S. O. Rice, "Statistical properties of a sine wave plus random noise," Bell Syst. J., vol. 27, pp. 109-157, Jan. 1948.

[4] R. S. Hoyt, "Probability functions for the modulus and angle of the normal complex variate," Bell Syst. J., vol. 26, pp. 318-359, Apr. 1947.

[5] M.Nakagami, "Them-distribution-A general formula of intensity distribution of rapid fading," in StatisticalMethods in RadioWave Propagation, W. G. Hoffman, Ed. Oxford, U.K.: Permagon Press, 1960, pp. 3-36.
[6] M.A. Taneda, J. Takada, and K. Araki, "A new approach to fading:Weibull model," in Proc. IEEE Int. Symp. Pers. Indoor Mobile Radio Commun., Osaka, Japan, Sep. 1999, pp. 711-715.

[7] F. Babich and G. Lombardi, "Statistical analysis and characterization of the indoor propagation channel," IEEE Trans. Commun., vol. 48, no. 3, pp. 455-464, Mar. 2000.

[8] J.B. Andersen, Statistical distributions in mobile communications using multiple scattering, in: Proceedings of the General Assembly of the International Union of Radio Science, Maastricht, The Netherlands, 2002.

[9] V. Erceg, S.J. Fortune, J. Ling, A. Rustako, R. Valenzuela, Comparisons of a computer-based propagation prediction tool with experimental data collected in urban microcellular environments, IEEE J. Sel. Area Comm. 15(4) (1997) 677-684.

[10] D. Chizhik, G. J. Foschini, M. J. Gans, and R. A. Valenzuela, "Keyholes, correlations, and capacities of multielement transmit and receive antennas," IEEE Trans. Wireless Commun., vol. 1, no. 2, pp. 361-368, Apr. 2002.

[11] H. Shin and J. H. Lee, "Performance analysis of space-time block codes over keyhole Nakagami-m fading channels," IEEE Trans. Veh. Technol., vol. 53, no. 2, pp. 351-362, Mar. 2004.

[12] G.K. Karagiannidis, T.A. Tsiftsis, R.K. Mallik, "Bounds of multihop relayed communications in Nakagamim fading," IEEE T. Commun. 54(1) 18-22, 2006.

[13] G. K. Karagiannidis, N.C. Sagias, P.T. Mathiopoulos, “ N*Nakagami: A Novel Stochastic Model for Cascaded Fading Channels," IEEE Transactions on Communications, vol. 55, no.8, August 2007.

[14] N.C. Sagias, G.C. Tombras, “ On the Cascaded Weibull fading channel model," Journal of the Franklin Institute, 344(2007) 1-11, July 2006.

[15] G. K. Karagiannidis, "Moments-based approach to the performance analysis of equal gain diversity in Nakagami-m Fading," IEEE Trans.Commun., vol. 52, no. 5, pp. 685-690, May 2004.

[16] M. H. Ismail and M.M.Matalgarh, "Performance of Dual Maximal Ratio Combining Diversity in Nonidentical Correlated Weibull fading Channels using Padé Approximation," IEEE Transaction on communication, vol. 54, no. 3, March 2006.

[17] H. Amindavar and J. A. Ritcey, "Padé approximation of probability density functions," IEEE Transactions on Aerospace and Electronic Systems, vol. 30, no. 2, pp. 416-424, 1994.

[18] J. W. Stokes and J. A. Ritcey, "A general method for evaluating outage probabilities using Padé approximations," in Proc. IEEE Global Telecommun. Conf., vol. 3, Sydney, Australia, pp. 1485-1490, Nov.1998.

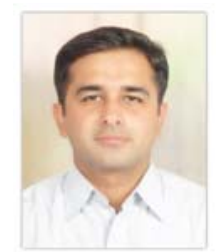

Jyoteesh Malhotra received the B.Eng. degree (with Distinction) in Electronics and Telecommunication from the Amravati University, India, in 1994, M.Tech. degree (with Distinction) in Electronics \& Communication from Panjab Technical University, India, in 2001. From 1994 to 2006 he worked with MCP College, DAV Institute of Engineering and Technology, Jalandhar, and Panjab University, Chandigarh as lecturer. Currently, he is working as lecturer in ECE dept. at Guru Nanak Dev University Regional Campus, Jalandhar and working towards his $\mathrm{PhD}$ degree. His current research includes statistical modeling $\&$ simulation of wireless fading channels and diversity techniques. He is a life member of the Indian Society of Technical Education (I.S.T.E.), New Delhi. 


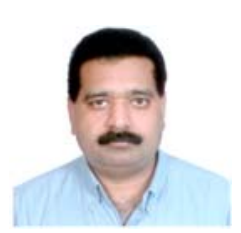

Ajay $\mathbf{K}$ Sharma received his $\mathrm{BE}$ in Electronics and Electrical Communication Engineering from Panjab University Chandigarh, India in 1986, MS in Electronics and Control from Birla Institute of Technology (BITS), Pilani in the year 1994 and $\mathrm{PhD}$ in Electronics Communication and Computer Engineering in the year 1999. His $\mathrm{PhD}$ thesis was on "Studies on Broadband Optical Communication Systems and Networks". From 1986 to 1995 he worked with TTTI, DTE Chandigarh, Indian Railways New Delhi, SLIET Longowal and National Institute of technology (Erstwhile Regional Engineering College), Hamirpur HP at various academic and administrative positions. He has joined National Institute of Technology (Erstwhile Regional Engineering College) Jalandhar as Assistant Professor in the Department of Electronics and Communication Engineering in the year 1996. From November 2001, he has worked as Professor in the same department and presently he is working as Professor in Computer Science \& Engineering in the same institute. His major areas of interest are broadband optical communication systems, dispersion compensation, fiber nonlinearities, optical soliton transmission, WDM systems and networks, Radio-over-Fiber (RoF) and wireless communication and networks. He has published 185 research papers in the International/National Journals/Conferences and 12 books. He has supervised $9 \mathrm{PhD}$ and 15 M.Tech theses. He has completed two R\&D projects funded by Government of India and one project is ongoing. Presently he is associated to implement the World Bank project of 209 Million for Technical Education Quality Improvement programme of the institute. He is technical reviewer of reputed international journals like: Optical Engineering, Optics letters, Optics Communication, Digital Signal Processing. He has been appointed as member of technical Committee on Telecom under International Association of Science and Technology Development (IASTD) Canada for the term 2004-2007 and he is Life member of Indian Society for Technical Education (I.S.T.E.), New Delhi.

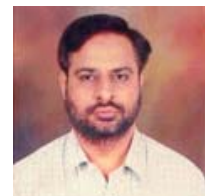

R. S. Kaler was born in Kausoli, Himachal Pradesh, India, on 31 December 1968. He obtained his bachelor's degree in Electronics Engineering with distinction from the Department of Electronics Technology, Guru Nanak Dev University, Amritsar, India, and Master's degree in Electronics Engineering from Panjab University, Chandigarh, India. He obtained his Ph.D. degree from Panjab Technical University, Jalandhar, in 2003. He worked at Panjab Communication Limited and Electronics Systems Panjab Limited, Mohali, from 1990 to 1994. He then joined BBSEC Fatehgarh Sahib as a lecturer and became an Assistant Professor in 1998. In 1999, he joined Sant Longowal Institute of Engineering and Technology, Longowal, Punjab, India as Assistant Professor in the Department of Electronics and Communication Engineering and continued till 2003. In 2003, he joined as Professor in Electronics and Communication Engineering Department at Thapar Institute of Engineering and Technology (Deemed University), Patiala. Presently he is working as Professor and Head of the same department. His present interests are fiber dispersion, nonlinearities and mobile communication. He has over 100 research papers out of which 40 are in international/national journals and rests are in international and national conferences. He has two R\&D projects and has organized many conferences. He is a life member of the Institution of Engineers (India) and Indian Society of Technical Education. 